\title{
La enseñanza del Periodismo en la Escuela Oficial franquista (1941-1975) analizada por sus exalumnos
}

\author{
Journalism teaching at the Franquist Official School (1941-1975) analyzed \\ by its former students
}

\author{
Dra. Pilar Sánchez-García \\ Universidad de Valladolid | Plaza del Campus Universitario, s/n, 47011 Valladolid | España | \\ http://orcid.org/0000-0002-6223-182X | pilar.sanchez@uva.es
}

\section{Dra. Marta Redondo García}

Universidad de Valladolid | Plaza del Campus Universitario, s/n, 47011 Valladolid | España | http://orcid.org/0000-0001-8143-465X| marta.redondo@hmca.uva.es

\author{
Alba Diez-Gracia \\ Universidad de Valladolid | Plaza del Campus Universitario, s/n, 47011 Valladolid | España | \\ http://orcid.org/0000-0001-9262-3771| alba.diez.gracia@alumnos.uva.es
}

Fechas | Recepción: 29/03/2021 | Aceptación: 05/08/2021

\section{Resumen}

La investigación tiene como objetivo profundizar en la formación que recibieron los periodistas durante la Dictadura franquista a través de la Escuela Oficial de Periodismo (1941-1975). Emplea una doble metodología de revisión documental y entrevista a fuentes primarias acerca de tres categorías: influencia ideológica, calidad formativa y ascendiente en el paso a la Universidad. Se obtiene el testimonio de exalumnos de la EOP en sus tres sedes: Madrid, Barcelona y La Laguna, entre ellos Diego Carcedo, Luis María Ansón o Juan Cruz. La investigación propone un análisis en tres etapas: control férreo formativo y colegial (1941-1958); apertura a iniciativas privadas (1958-1966) y preuniversitaria (1969-1975). Una propuesta cronológica que es secundada por el testimonio de los entrevistados que evidencian cómo los intentos de adoctrinamiento que marcaron la Escuela en la primera mitad de su existencia fracasaron en la segunda ante un alumnado en buena medida

\section{Abstract}

The purpose of this research is to examine how journalists were trained during the Franco dictatorship in the Official School of Journalism (1941-1975). It combines two methodologies: document analysis and interviews with primary sources about three categories: ideological influence in education; quality in training and boost or discourage to University studies. Former EOP students in Madrid, Barcelona and and gathered testimonies of journalists such as Diego Carcedo, Luis María Ansón or Juan Cruz. The research offers an analysis in three periods: formative and collegial control (1941-1958); opening to private initiatives (1958-1966) and pre-university stage (1969-1975). This chronological proposal is supported by the testimony of the consulted students who say that, despite its ideological origin, the attempts of indoctrination at EOP failed, mostly because students were refractory in the second half of its existence. The students describe the poor technical resources at the EOP and stand out 
refractario. Los alumnos describen los limitados recursos técnicos de la EOP, aunque valoran la capacitación profesional de los estudios y el contacto que permitían con los medios de comunicación a través de las prácticas. Finalmente interpretan que la Escuela supuso un impulso más que una rémora en el paso de los estudios de Periodismo hacia la formación superior.

Palabras clave: periodismo, franquismo, formación de periodistas, Escuela Oficial de Periodismo, EOP. the practical training of the studies. Finally, they consider that the School was an impulse towards higher education.

\section{INTRODUCCIÓN}

La enseñanza del Periodismo en España abarca un siglo de cambios y evolución. La mirada histórica a las diferentes etapas formativas aporta una relevante visión acerca de la influencia que diferentes grupos de poder -Iglesia, sindicatos, empresarios, gobiernos- han intentado ejercer en la formación de los periodistas a través de iniciativas docentes en toda la etapa preuniversitaria (1926-1971). Un recorrido por los hitos principales permite ahondar en la influencia ejercida en la etapa franquista durante más de tres décadas a través de la Escuela Oficial de Periodismo (EOP, 1941-1975) y el Registro Oficial de Periodistas. Dos eslabones que constituyen una forma de control informativo (Humanes, 1997) de un trabajo considerado entonces un oficio o un arte innato, más que una profesión, vinculado a la idea de la prensa como industria (Aguinaga, 1984; Mainar, 2005).

La Escuela franquista evoluciona durante tres décadas hasta que en 1971 el Periodismo pasa a la Universidad y llega a sumar 40 facultades con estos estudios en el curso 2019-20 (SánchezGarcía et al., 2019). En todo este recorrido, la literatura académica ha mostrado interés en la enseñanza periodística en España hilando un relato casi por décadas (Altabella, 1979; Moragas, 1981; Aguirre, 1988; Vigil y Vázquez, 1987; Gordon, 1991; Humanes, 1997; Rodrigo, 2001; Videla, 2002; Real 2004; Tejedor, 2006; Salaverría y Barrera, 2009; Sánchez-García, 2014 y 2016). Sin embargo, resultan menos prolíficas las investigaciones centradas exclusivamente en la propia EOP (Altabella, 1971) y las que existen reflejan un debate dicotómico, aún no cerrado, en torno a su impulso profesionalizante (Aguinaga, 1984) o la rémora formativa que representa en el paso a la Universidad (Humanes, 2002). Así, frente a quienes únicamente ven en la EOP un interés dictatorial por controlar ideológicamente la información, vinculada al uso de los medios como herramienta intervenida destinada al control y la propaganda (Sevillano, 1998), se contrapone el argumento de quienes destacan los logros de la Escuela franquista porque "desbrozó el sendero y superó ella sola unas etapas sin las que no se abriría ante nosotros un panorama tan esperanzador para la enseñanza del periodismo en España" (Benito, 1967, p. 11). Un debate que la presente investigación trata de abordar recopilando por primera vez el testimonio de antiguos estudiantes de la EOP en sus tres sedes.

A partir de los estudios precedentes y la consulta a fuentes primarias, esta investigación surge con el objetivo principal de analizar la evolución formativa de los periodistas durante el franquismo a través de la Escuela Oficial de Periodismo y con dos objetivos secundarios: 
O1. Establecer etapas que diferencien la evolución de la EOP en tres décadas marcadas por los cambios de las normativas y el control ideológico y/o profesionalizante en la formación de los estudiantes.

O2. Reunir el testimonio de alumnos de la EOP sobre la formación recibida diferenciando tres ejes: la visión ideológica, la calidad de la formación y la influencia en el paso a los estudios universitarios.

Sus propios alumnos ayudan a tejer en esta investigación el relato de sus 30 años de historia.

\subsection{Evolución de la EOP en tres etapas formativas (1941-1971)}

Varios estudiosos (Moragas, 1981; Rodrigo, 2001) coinciden en establecer etapas, desde el punto de vista informativo, durante la Dictadura: el control de la información y la propaganda inicial (1939-1945); el fin de la Segunda Guerra Mundial y el rechazo internacional a los fascismos (1946-1966) y el auge de la investigación y formación en Comunicación en el aperturismo informativo del tardofranquismo (1966-1976). A través de estos hitos y del análisis de investigaciones precedentes se propone aquí analizar la evolución formativa de los periodistas en la EOP por etapas, tomando también como referencia el planteamiento de Humanes (2002) con una periodificación similar, aunque, en su caso, más centrada en el cambio de planes de estudio. En la presente propuesta se analiza el control formativoinformativo a partir de dos variables principales: los sucesivos cambios normativos que rigen la EOP y la apertura a otras iniciativas que flexibilizan el control formativo. Así, se proponen tres etapas con los hitos que marcaron cada una de ellas (Tabla 1):

- $\quad$ Control férreo formativo y colegial (1941-1958)

- $\quad$ Apertura a otras iniciativas formativas privadas (1958-1966)

- $\quad$ Etapa pre-universitaria (1969-1975)

Tabla 1

Hitos en la enseñanza de la Escuela Oficial de Periodismo y sus precedentes (1897-1975)

\begin{tabular}{|c|c|}
\hline \multirow{6}{*}{ 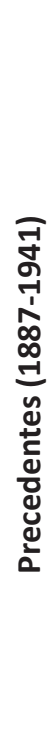 } & $\begin{array}{l}\text { 1887: Primer curso organizado en España por el periodista Fernando Araujo en la } \\
\text { Universidad de Salamanca }\end{array}$ \\
\hline & $\begin{array}{l}\text { 1915: El Congreso Nacional de Prensa pide que Periodismo se convierta en una } \\
\text { carrera universitaria }\end{array}$ \\
\hline & $\begin{array}{l}\text { 1926: Durante la dictadura de Primo de Rivera, abre sus puertas la Escuela de El } \\
\text { Debate ligada al periódico homónimo e impulsada por su director, el sacerdote y } \\
\text { periodista Ángel Herrera Oria }\end{array}$ \\
\hline & $\begin{array}{l}\text { 1936: Guerra Civil española. Cierre de la Escuela de El Debate y organización de } \\
\text { cursos propagandísticos }\end{array}$ \\
\hline & $\begin{array}{l}\text { 1937: Seminario nacional sindicalista de Estudios Periodísticos organizado por el } \\
\text { bando nacional }\end{array}$ \\
\hline & $\begin{array}{l}\text { 1938: Escuela de Mundo Obrero impulsada desde el diario comunista Mundo } \\
\text { Obrero } \\
\text {-Las Juventudes Socialistas unificadas organizan en Madrid un 'Cursillo de } \\
\text { Periodismo de guerra' }\end{array}$ \\
\hline
\end{tabular}




\begin{tabular}{|c|c|}
\hline & $\begin{array}{l}\text { 1938: Ley de Prensa dispone "la organización académica del periodismo" y el } \\
\text { Registro Oficial de periodistas }\end{array}$ \\
\hline \multirow{21}{*}{ 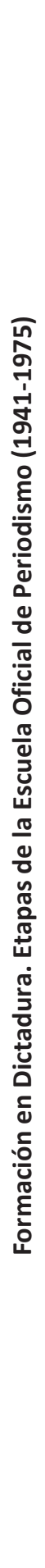 } & PRIMERA ETAPA (1941-1958) \\
\hline & $\begin{array}{l}\text { 1940: La Dirección General de Prensa, dependiente de la Subsecretaría de Prensa y } \\
\text { Propaganda del Ministerio de la Gobernación, organiza cursos con la colaboración } \\
\text { de la Agencia EFE }\end{array}$ \\
\hline & $\begin{array}{l}\text { 1941: La Delegación Nacional de Prensa y Propaganda aprueba la creación de la } \\
\text { EOP }\end{array}$ \\
\hline & $\begin{array}{l}\text { 1942: El } 2 \text { de enero comienzan las clases oficiales de la EOP en la calle Ayala, } \\
\text { número } 5 \text { de Madrid }\end{array}$ \\
\hline & $\begin{array}{l}\text { 1951: Se crea el Ministerio de Información y Turismo del que pasa a depender la } \\
\text { EOP }\end{array}$ \\
\hline & 1952: La EOP abre de manera temporal su delegación en Barcelona, hasta 1963 \\
\hline & $\begin{array}{l}\text { 1954-55: Ante el aumento de estudiantes, la EOP endurece las pruebas de acceso y } \\
\text { pide tesina final }\end{array}$ \\
\hline & SEGUNDA ETAPA (1958-1966) \\
\hline & 1958: La Iglesia crea el Instituto de Periodismo de Navarra \\
\hline & $\begin{array}{l}\text { 1960: Se inaugura la Escuela de Periodismo de la Iglesia, fundada por Ángel Herrera } \\
\text { Oria. Posteriormente abrirá delegaciones en Barcelona y Valencia. }\end{array}$ \\
\hline & 1962: Abre sus puertas la Escuela Oficial de Cinematografía \\
\hline & $\begin{array}{l}\text { 1963: La EOP exige a sus estudiantes el título preuniversitario para acceder a sus } \\
\text { estudios }\end{array}$ \\
\hline & $\begin{array}{l}\text { 1964: La EOP abre en la Universidad de La Laguna / Se inaugura la Escuela Oficial de } \\
\text { Publicidad }\end{array}$ \\
\hline & 1967: Se inaugura la Escuela Oficial de Radiodifusión y Televisión \\
\hline & $\begin{array}{l}\text { 1968-69: Reapertura de la delegación de la EOP en Barcelona con personalidad } \\
\text { propia }\end{array}$ \\
\hline & TERCERA ETAPA (1966-1975) \\
\hline & $\begin{array}{l}\text { 1966: Aprobación de la Ley de Prensa que flexibiliza la libertad informativa y } \\
\text { profesional. } \\
\text { La EOP celebra sus } 25 \text { años reorganizando su plan de estudios con la dirección de } \\
\text { Juan Beneyto }\end{array}$ \\
\hline & $\begin{array}{l}\text { 1967: Orden ministerial que decreta el título superior de periodista con } 4 \text { años de } \\
\text { carrera }\end{array}$ \\
\hline & $\begin{array}{l}\text { 1969: Cambio de reglamento de la EOP para reconocer a los titulados de las } \\
\text { escuelas privadas }\end{array}$ \\
\hline & $\begin{array}{l}\text { 1970: Ley General de Educación que dispone la incorporación de los estudios de } \\
\text { Periodismo a la Universidad y establece el cierre de las escuelas (1975) }\end{array}$ \\
\hline & $\begin{array}{l}\text { 1971-72: Los estudios de Periodismo se ofrecen en la Universidad Complutense, } \\
\text { Universidad Autónoma de Barcelona y Universidad de Navarra }\end{array}$ \\
\hline
\end{tabular}




\begin{tabular}{|l|l|}
\hline $\begin{array}{l}\text { 1975: Cierre de la Escuela Oficial de Periodismo tras la formación durante } 35 \text { años } \\
\text { de } 1.927 \text { periodistas }\end{array}$
\end{tabular}

Fuente: elaboración propia.

\subsubsection{Primera etapa: control formativo y colegial (1941-1958)}

La primera etapa es la más larga y se corresponde con el período más restrictivo de la Dictadura en la formación de periodistas (1941-1958). Una fase solapada con la etapa de construcción del aparato propagandístico del régimen franquista, que comienza a partir de 1938 con la creación de diversos medios como la Agencia EFE o la Cadena de Prensa del Movimiento, y que continuaría con la cristalización de un nuevo orden informativo controlado y monopolizado por la dictadura (Sevillano, 1998).

El embrión de la apertura de la EOP son unos cursos organizados en 1940 por la Dirección General de Prensa que surgen ante la falta de periodistas titulados para inscribirse en el Registro Oficial regulado por la Ley de Prensa de 1938. Tras esta experiencia, en noviembre de 1941 el Gobierno aprueba la creación de la Escuela dependiente de la Delegación Nacional de Prensa y Propaganda, órgano considerado como el 'aparato' censor del Régimen, dirigido por Juan Aparicio, y que, "junto al sindicato vertical y los tribunales especiales, tratarían de controlar el acceso y ejercicio de la profesión" (Luján, 2014). En ese momento, la doctrina franquista sobre medios de comunicación "consideraba al periodista como un funcionario más del Estado" (Beneyto, 1944).

Las clases de la Escuela Oficial de Periodismo (EOP) comienzan el 2 de enero de 1942, en la calle Ayala número 5 de Madrid $^{1}$. Su apertura entierra la idea primigenia de que la Escuela forme parte de secciones de Periodismo dentro de las Facultades de Filosofía y Letras (establecida en la citada Ley de 1938) y se convierte en la única vía de acceso para ejercer el periodismo. Juan Aparicio, entonces director general de Prensa, se rodea de un elenco de profesores, como Bartolomé Mostaza, que, como él, habían trabajado en la escuela de El Debate (Videla, 2002) y de profesionales en activo en su mayoría. El control ideológico del profesorado era un elemento fundamental de esta primera etapa en la que la EOP depende del Ministerio de la Secretaría General del Movimiento. Así, es el Ministerio de Información quien nombra en esta época al director del centro, y el Centro Directivo, conformado por personalidades de la administración pública, las agrupaciones profesionales y representantes mediáticos, haciendo patente una conexión entre el ámbito formativo y empresarial (Beneyto, 1958). Igualmente, el decreto del 29 de julio de 1939 obliga a los profesores a adaptar su enseñanza al dogma, a la moral y al Derecho Canónico (Tapia, 2011).

Para ingresar en la Escuela se tenía que estar en posesión del título de Bachiller, Maestro u otro análogo y ser militante de Falange Española Tradicionalista y de las Jons (ABC, 1941). "Este último filtro pretendía vedar el ejercicio del Periodismo a cualquier elemento ajeno al sistema" (Videla, 2002, pp. 146-147). El número de alumnos queda fijado en veinte, aunque la llegada de estudiantes de otras partes del país lleva a abrir delegaciones en otros lugares de España, como serán las delegaciones de Barcelona y La Laguna (Beneyto, 1958).

\footnotetext{
${ }^{1}$ Posteriormente se ubicó en otras sedes como en un chalé de la calle Monte Esquinza y en la sede del Ministerio de Información y Turismo (entrevista con Luis Ángel de la Viuda, 15-12-2017).
} 
Al inicio de esta primera etapa existen algunos intentos de trasladar la formación periodística a la Universidad. El 11 de abril de 1946, cuando la Escuela pasa a depender del Ministerio de Educación Nacional, el entonces ministro Ibáñez Martín anuncia ante una comisión de titulados de la EOP la decisión de insertarla en la enseñanza superior mediante la aprobación de una nueva Ley que se estaba fraguando. Como nuevo director general de Prensa, pasa a dirigir la EOP Tomás Cerro Corrochano, procedente de la extinta Escuela de El Debate, quien paraliza la actividad de la Escuela dos años, de 1947 a 1949, y la sustituye por la convocatoria de cinco nuevos cursillos intensivos para dar cabida a quienes ya eran profesionales del periodismo y que tenían que adjuntar el documento que acreditara plena adhesión al Movimiento y su dedicación profesional. "Fue un bache, una etapa oscura que dejó moribunda a la Escuela porque lo que se buscaba era dar el título a manta a los que ejercían la profesión sin título" (Aguinaga, entrevista 2018). Finalmente se paraliza la opción universitaria y se reanudan las clases en la EOP que pasa a depender del Ministerio de Información y Turismo en 1951.

En cuanto a las asignaturas que comienzan a impartirse resultan un compendio de materias referidas a cultura general, doctrina política nacional-sindicalista y las propias del ejercicio periodístico: Redacción Literaria, Historia del Periodismo, Legislación de Prensa, Información y Reportajes, Titulación, y Confección y Tipografía. La formación se completa con la realización de los periódicos en la misma Escuela Ilamados España una, España grande y España libre (Aguinaga, entrevista 2018; Humanes, 2002). El progresivo aumento de solicitudes de ingreso obliga a realizar duros controles de acceso. A partir del curso 1954-55 la Escuela exige a los interesados un dominio de la actualidad nacional y extranjera, además de mostrar conocimientos de francés e inglés.

Una vez completados los cursos, los alumnos pasan por pruebas orales y escritas para graduarse. Deben redactar un artículo de fondo, seleccionar titulares y subtitular una serie de noticias de agencias o rehacer un reportaje, entre otras tareas. Por su parte, el examen oral aborda cuestiones de organización de las empresas, legislación y lenguas extranjeras. Esta prueba se realiza ante un jurado en el que "figuran miembros de la dirección y del cuerpo docente, pero el papel principal lo desempeñan dos directores de diarios que representan los intereses del gremio" (Beneyto, 1958, p. 201). Desde 1954 se incluye la obligatoriedad de realizar un trabajo de fin de estudios o tesina.

Es entonces cuando los estudiantes aprobados reciben su diploma de periodista, requisito imprescindible para inscribirse en el Registro Oficial que les da derecho a ejercer inmediatamente la profesión. De la EOP salen los primeros periodistas titulados en España con reconocimiento oficial, cuya formación y colegiación dependen exclusivamente del Estado. Este título formativo "aparece como el instrumento básico para la creación y mantenimiento de la profesionalidad del Periodismo en ejercicio, en cuanto que la Escuela viene a crear la titularidad del periodista" (Aguinaga, 1984, p. 138).

El carné, expedido por la Delegación Nacional de Prensa, les diferenciaba de los profesionales de la República sin colegiar. "La consecución del carnet profesional obligatorio era tan difícil para los periodistas que habían trabajado en zona republicana como en la metáfora bíblica: que un camello pasase por el ojo de una aguja" (Núñez, 1997, p. 206). Una época de control informativo férreo que la misma autora denomina 'el viacrucis del profesional apolítico'. 
Desde la dirección del Ministerio de Información y Turismo se refuerzan a partir de mediados de los años 50 los cimientos de la Escuela que comienza a reducir la carga política en sus enseñanzas (Beneyto, 1957). Estos cambios, unidos a las modificaciones de los planes de estudio, dejan vislumbrar cómo en las décadas siguientes la Escuela "lentamente va consiguiendo una mayor profesionalidad en detrimento de su excesiva politización inicial" (Real, 2004, p. 483).

\subsubsection{Segunda etapa. Expansión y apertura a otras escuelas privadas (1958-1966)}

La segunda etapa coincide con una apertura de la formación periodística a la iniciativa privada al permitir el funcionamiento de otros centros adscritos a la EOP. El primer ejemplo es el Instituto de Periodismo de Navarra que se crea en 1958, por iniciativa de la Iglesia (Barrera, 2009a). Le sigue la Escuela de Periodismo de la Iglesia que en 1960 refunda Ángel Herrera Oria y que años después abre delegaciones en Barcelona y Valencia. Estos dos centros privados y católicos conviven con la EOP que, a su vez, estrena delegaciones en Barcelona y La Laguna. Todas ellas bajo las prescripciones de la Escuela Oficial de Madrid que no pierde su control sobre los programas y la convalidación de títulos. A pesar del control "la aparición y el progresivo enraizamiento de las escuelas no oficiales, supusieron un mayor pluralismo en el horizonte de la educación del periodista, y también un inicio de competencia" (Beneyto, 1958, p. 202).

En el devenir formativo de las tres escuelas juega un papel relevante el aperturismo informativo de la Ley de Prensa e Imprenta de 1966 (Barrera, 2002b) que representa un punto de inflexión con la eliminación de la censura previa y que regula el campo de la empresa periodística mediante requisitos de carácter más aperturista, lo que en palabras de Ángel Benito supone "una consecuencia de la tensión vital hacia la libertad" que la sociedad española exigía a la administración” (1967, p. 16).

A partir de 1963, la EOP exige a los alumnos el título preuniversitario, lo que, en la práctica, eleva a la Escuela al ámbito de la enseñanza superior. El programa de estudios se prolonga tres años (entre 1951 y 1967) mientras la demanda de alumnos no deja de crecer. En todo este tiempo, la EOP abre nuevas delegaciones en Barcelona y Canarias. Aunque la primera sección dependiente de la de Madrid abre de forma temporal en Barcelona en 1952 a través de cursos trimestrales (Videla, 2002), suspende su actividad en 1963 y reabre nuevamente en 1968 con personalidad propia y patronato autónomo. Por orden ministerial del 6 de septiembre de ese año, el ministro de Información, Fraga Iribarne, autoriza la Escuela Oficial de Periodismo de Barcelona y el 9 de enero de 1969 abre sus puertas, bajo la dirección de Julio Manegat. Comienza con el plan de estudios dependiente de Madrid, pero, poco a poco, logra su autonomía en el programa, en la firma de títulos y en la inscripción en el Registro Oficial de Periodistas (Videla, 2002).

En el caso de Canarias, la EOP abre su sede en 1964 en la Universidad de La Laguna con una sección propia de Periodismo reconocida por Orden Ministerial de Información y Turismo de 30 de noviembre de ese año, aunque llevaba funcionado desde final de 1963 (Acirón, 1988). La mezcla de profesorado de diferentes disciplinas universitarias junto con profesionales de los medios de comunicación lleva a defender desde dentro de la Universidad, que la sección de Periodismo de La Laguna tiene carácter universitario y está considerada como "la pionera de las universidades públicas españolas en acoger en su seno los estudios de Ciencias de la 
Información y la Comunicación y, por tanto, de reconocerle al Periodismo su identidad de ciencia experimental, de raíz social y humanística" (Acirón 1988, p. 2).

\subsubsection{Tercera etapa. Del aperturismo dictatorial a las aulas universitarias (1966-1975)}

La Escuela Oficial de Periodismo celebra su 25 cumpleaños recogiendo los resultados de la reorganización realizada en la etapa anterior, que permite su consolidación formativa. Uno de los artífices fue Juan Beneyto, autor de un informe sobre la Escuela encargado por la Unesco (1958) en el que restaba peso a la visión de politización en favor del carácter formativo de la EOP. Como director -el primero de la Escuela que no ocupa la Dirección General de Prensa (Humanes, 2002)- había logrado imprimir "un carácter de gran seriedad académica y social al exigirse los mismos títulos que la Universidad pide a quienes van a cursar estudios en sus facultades" (Videla, 2002, p.151).

En esta tercera etapa toma el relevo de la dirección Bartolomé Mostaza y los planes de estudio pasan a durar cuatro años desde 1967, renovados en 1969 con una amplia variedad de contenidos periodísticos y de cultura general. Un cambio significativo llega con la orden ministerial de 20 de abril de 1967 que decreta que el de periodista constituye un título de carrera superior con el plan de estudios preparado por una comisión especial (Vigil, 1987). Dicha Orden ministerial establece en su preámbulo que la EOP tiene dos objetivos, formar profesionales y teóricos de la información (Humanes, 1997). Es entonces cuando la EOP implanta pruebas más rigurosas de entrada y de salida. Sustituye el cuestionario de ingreso por la aprobación mencionada del preuniversitario, una memoria autobiográfica, un ejercicio psicotécnico y un cuestionario sobre temas de actualidad; y publica las condiciones del nuevo examen de Grado final que incluye pruebas prácticas y teóricas orales y escritas, junto con la presentación de una tesina.

Aunque se sigue conviviendo con la censura informativa y la autocensura en el tardofranquismo (Barrera, 2002b), con la década de los años setenta llegarán cambios irreversibles en la sociedad y en la EOP, impulsados por el desarrollismo o los movimientos de oposición estudiantil y obrera, vinculados a movimientos sociales (Baldó, 2008; 2016). El endurecimiento en la exigencia de las pruebas de acceso y graduación, la ampliación de los programas y la incorporación de 'teóricos' representan los preparativos claros del paso del Periodismo a la Universidad que se hace efectivo en 1970 con la Ley General de Educación que determina la clausura de las escuelas de Periodismo en diciembre de 1975 después de treinta y cuatro años de funcionamiento. Bajo la dirección de Emilio Romero, artífice final del salto a la Universidad, la Escuela Oficial de Periodismo cierra sus puertas (Gordon, 1991) después de haber formado a 25 promociones ordinarias y once promociones especiales de periodistas.

\section{METOdOLOGÍA}

El estudio se realiza con una doble metodología cualitativa de revisión bibliográfica y de acercamiento a fuentes primarias. En primer lugar, se realiza una revisión documental de estudios precedentes relacionados con la enseñanza periodística durante el franquismo desde una perspectiva histórico-social (Aróstegui, 1995). Incluye la búsqueda presencial en fondos originales como el archivo documental de la Asociación de la Prensa de Madrid, el acercamiento al fondo bibliográfico personal del secretario y profesor de la EOP Enrique de Aguinaga completada con entrevistas en profundidad al citado profesor y periodista. Esta 
metodología permite revisar los estudios precedentes estableciendo etapas diferenciadas en las tres décadas de la EOP (O1) a través de las sucesivas modificaciones normativas y formativas de esta etapa pre-científica del Periodismo (Fernández Del Moral, 1991), articulando el marco teórico anteriormente expuesto.

En segundo lugar, la investigación recurre al método de la encuesta a fuentes primarias: profesores y alumnos de la EOP, como individuos y sujetos históricos (Sanz-Hernández, 2005). Una técnica sustentada en las fuentes orales ampliamente utilizada como instrumento de análisis en otras investigaciones del periodo estudiado (Díaz-Sánchez y Gago-González, 2006; Ramos y Studer, 2012) que aporta al relato histórico "la materialización de una experiencia, un testimonio, una mirada capaz de contar los cambios, las condiciones socioculturales de una época (...) y, sobre todo, permite comparar con fuentes documentales" (Eiora, 2014, p. 258).

Se diseñó un cuestionario estandarizado compuesto por doce preguntas abiertas con el fin de recabar las respuestas de los participantes "en toda su complejidad (...) utilizando sus palabras, en los términos que consideren más adecuados y sin condicionamientos previos" (Meneses y Rodríguez-Gómez, 2011). Las preguntas se organizaron en cuatro bloques para dar respuesta sistematizada a las tres categorías a analizar: influencia ideológica, calidad formativa y ascendiente de la EOP en el paso a la Universidad (ver tabla 3).

Los encuestados fueron localizados gracias a un muestreo en cadena o 'bola de nieve', una técnica especialmente aconsejable cuando la muestra es pequeña, está limitada y resulta difícil de hallar. Así, a las fuentes iniciales se les pidió que aportasen nuevos contactos de excompañeros de las tres delegaciones, Madrid, Barcelona y La Laguna (Tenerife), hasta localizar a más de una veintena de participantes, de los que finalmente se obtuvieron 17 respuestas (ver Tabla 2). Dado lo limitado de la muestra obtenida, los resultados no pueden ser estadísticamente representativos de la población objetivo. No obstante, entendemos que el relato de fuentes directas, nunca antes consultadas con el propósito de indagar en la enseñanza impartida en la EOP, aporta luz a la comprensión de esta etapa en la formación de los periodistas en España.

Tabla 2

Listado de ex alumnos de la EOP que participan en el cuestionario ${ }^{2}$

\begin{tabular}{|l|l|l|}
\hline Nombre del alumno & Delegación de la EOP & Año de promoción \\
\hline Enrique de Aguinaga & Madrid & $1944-1946$ \\
\hline Luis María Ansón & Madrid & $1954-1957$ \\
\hline Luis Ángel de la Viuda & Madrid & $1956-1959$ \\
\hline Miguel Ángel Gozalo & Madrid & $1958-1960$ \\
\hline Jesús Picatoste & Madrid & $1960-1964$ \\
\hline Homero Valencia & Madrid & $1963-1966$ \\
\hline Diego Carcedo & Madrid & $1965-1969$ \\
\hline Celso Almuiña & Madrid & $1970-1975$ \\
\hline Valentín Popescu & Barcelona & En torno a 1955 \\
\hline Antonio Merino & Barcelona & $1968-1972$ \\
\hline Amparo Moreno Sardá & Barcelona & $1970-1973$ \\
\hline
\end{tabular}

\footnotetext{
${ }^{2}$ En algunos casos el entrevistado no recordaba el año de ingreso o graduación o no lo ha hecho constar.
} 


\begin{tabular}{|l|l|l|}
\hline Manel López & Barcelona & $1970-1974$ \\
\hline Carles Guàrdia Moreno & Barcelona & $1970-1974$ \\
\hline Santiago Ramentol & Barcelona & $1968-1972$ \\
\hline Juan Cruz & La Laguna & $1967-1970$ \\
\hline Julio Pérez & La Laguna & $1968-1972$ \\
\hline Max Ebstein & La Laguna & ------ \\
\hline
\end{tabular}

Fuente: elaboración propia.

El cuestionario remitido está compuesto por 12 preguntas agrupadas en cuatro bloques ${ }^{3}$ (ver tabla 3): identificación de la promoción y sus características; presiones o consignas del régimen franquista en el aula; la percepción de la calidad de la formación recibida; y una valoración personal sobre si la EOP supuso una rémora o un impulso a la entrada de Periodismo en la Universidad.

\section{Tabla 3}

Estructura del cuestionario realizado a los exalumnos de la EOP

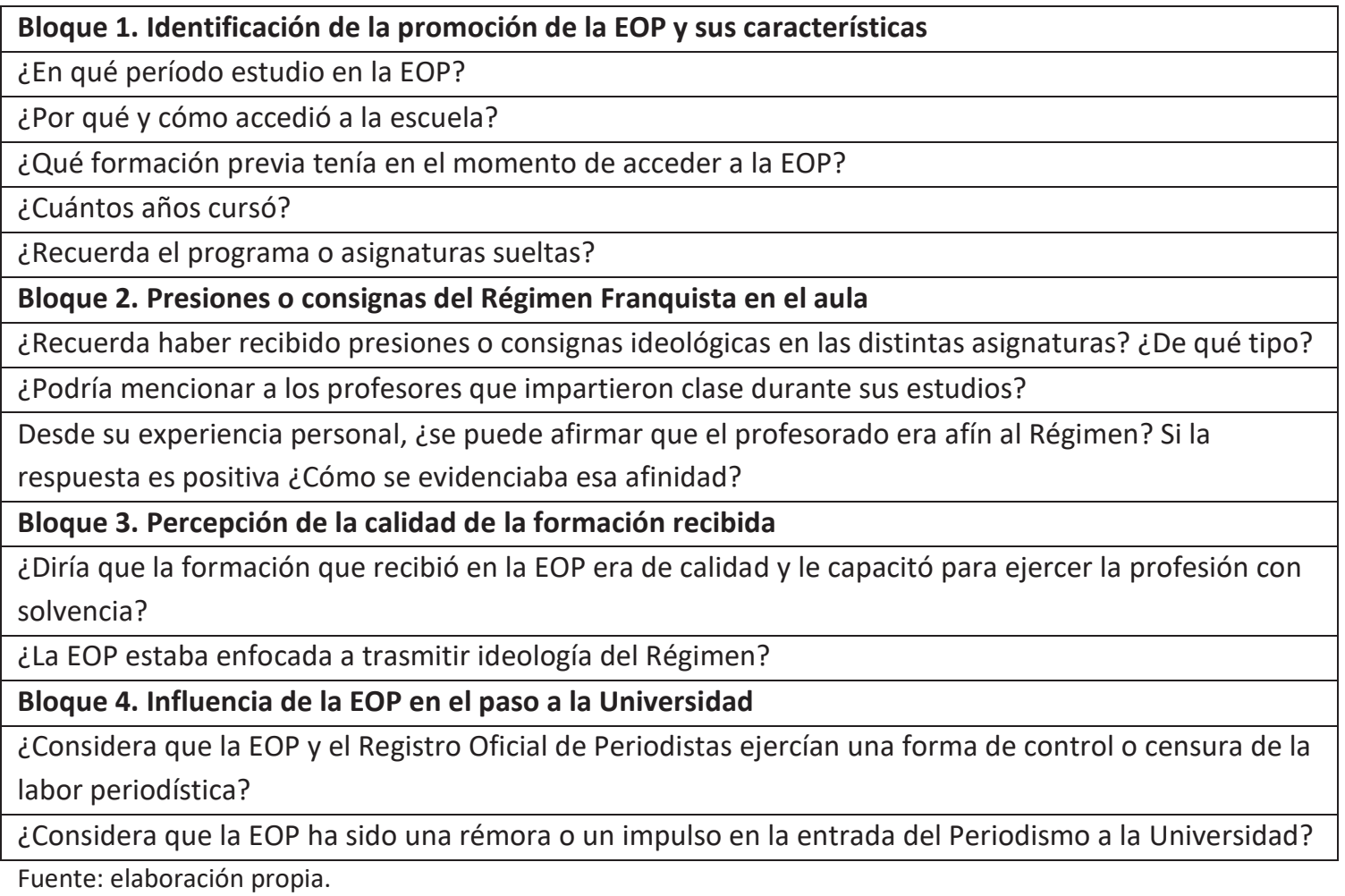

En torno a estas categorías de estudio se presentan ahora los resultados de la investigación en torno a los testimonios recogidos de los entrevistados.

\section{RESULTADOS}

\subsection{Fuentes primarias: exalumnos de la EOP de Madrid, Barcelona y Canarias}

El relato de la evolución de la EOP se completa ahora con la consulta a antiguos alumnos de las delegaciones de Madrid, Barcelona y La Laguna (Tabla 2) que permite comparar su experiencia en sus respectivos contextos y refrendar el análisis cronológico propuesto al localizar testigos

\footnotetext{
${ }^{3}$ Se remite vía mail entre noviembre de 2017 y septiembre de 2018 entre ex alumnos de la EOP en Madrid, Barcelona y Canarias. Se ha tenido la oportunidad de realizar la entrevista de forma presencial a Enrique de Aguinaga, Luis Ángel de la Viuda y Jesús Picatoste.
} 
de las tres etapas descritas aquí. A modo de ejemplo se señala que el primero en graduarse se corresponde con la quinta promoción, Enrique de Aguinaga ${ }^{4}$ (1944 y 1946), seguido de Luis María Ansón (1957), mientras que el último es Manel López (1974) graduado en la penúltima promoción de la Escuela. Sin embargo, existe predominancia de los alumnos pertenecientes a la última etapa (un $56,2 \%$ de los encuestados), seguida de la segunda (un $25 \%$ ), por lo que el retrato que abordan las encuestas es reflejo de la formación en la Escuela a lo largo de toda la década de los años 60 hasta su cierre en 1975.

\subsubsection{Visión ideológica: el adoctrinamiento en la formación de los periodistas de la EOP}

En las diferentes etapas de evolución de la EOP se detectan momentos de mayor o menor intento de adoctrinamiento ideológico dentro de un modelo de formación "intervencionista totalitario" (Pestano et al, 2011, p. 405) y así lo corroboran los exalumnos encuestados. En las respuestas se constata su convencimiento de que la Escuela nace como órgano de vigilancia formativa de los periodistas unido al control profesional ejercido a través del Registro Oficial. Luis Ángel de la Viuda (1956-1959) considera que el "pecado original" de la Escuela es que estaba diseñada para manejar directrices políticas: "El poder político montó una escuela para tener periodistas, no sé si controlados, pero sí identificados (...) No se puede engañar a nadie, era una escuela para registrar periodistas". Para Celso Almuiña (1970-1975) era una herramienta de registro de acceso a la profesión: "Basta ver la Ley 'provisional' de Prensa de 1938 de Serrano Súñer para darse cuenta del control de los periodistas. Si te quitaban el carné, que lo regalaban a los correligionarios, no podías trabajar en ningún medio".

Sin embargo, en la segunda y tercera fase que vivieron principalmente los alumnos encuestados, ese rigor inicial parece suavizarse. La mayoría descarta haber recibido presiones ideológicas, al menos explícitas, en las clases y, aunque gran parte del claustro de profesores había sido elegido precisamente por su afinidad y vinculación con el Régimen, algunos exalumnos recuerdan profesores no afines o que, incluso siéndolo, destacaban como docentes. Homero Valencia (1963-66) evoca la sutilidad con la que se manifestaba la afinidad al franquismo de los docentes: "no lo proclamaban en clase. Los alumnos lo sabíamos por sus biografías, trabajos profesionales, etc.". Apreciación parecida realiza Luis María Ansón (19541957): "En las asignaturas no había ni presiones ni consignas (...). Entre el profesorado, había de todo, aunque predominaba, por lo menos de forma externa, la afinidad al Régimen". Una vinculación al franquismo que no estaban reñida con su calidad como docentes:

Toda la carrera se ajustaba a las líneas políticas del Régimen y todos los profesores, con alguna excepción, actuaban fieles a aquellos principios (...) Pero el hecho de que fuesen del Régimen no quiere decir que todos fuesen desastrosos. Algunos eran buenos profesores o muy buenos. (Diego Carcedo, 1965-1969)

A medida que avanzan las promociones hacia el aperturismo y luego el tardofranquismo, los estudiantes dan por fracasado el fin ideológico en su formación y constatan un ambiente cada vez más contestatario entre el alumnado, coincidiendo con una etapa histórica que autores como Baldó (2008) denominan de "desarrollismo, tecnocracia y rebeldía" (2008, p. 55), caracterizada por el movimiento estudiantil y la protesta universitaria como oposición contra

\footnotetext{
${ }^{4}$ Fragmento de la entrevista realizada disponible en https://bit.ly/3yCotRe
} 
la dictadura, que empezó en 1955 y se radicalizó a partir de 1969 ante la continua represión de sindicatos y otras iniciativas.

En los primeros años parece que la Escuela estaba enfocada a transmitir ideología. En mi etapa se supone que también, pero sin mucho empeño ni mucho éxito. La mayor parte de los compañeros estábamos en contra, flirteábamos con organizaciones políticas y sindicales secretas, participábamos en manifestaciones universitarias y, eso también, procurábamos no salirnos del cauce oficial. (Diego Carcedo, 1965-1969)

Antonio Merino (1968-1972) califica de "prácticamente nula" la influencia ideológica de la Escuela en su etapa de estudiante: "La mayoría de estudiantes tenía formación marxista y existencialista, y los profes estaban desbordados por la dialéctica del momento".

En el caso de los alumnos de la sección de Barcelona, las respuestas constatan el declive de la Escuela como instrumento de propaganda que era palpable en los últimos años e incluso describen un ambiente más contestatario que el vivido en la EOP de Madrid.

Es posible que la Escuela se creara para fabricar periodistas adictos al régimen. Pero en Barcelona, no se respiraba este ambiente. El objetivo (si lo hubo) fracasó de forma contundente. (...) A casi nadie le interesaba la ideología del Régimen. Los profesores de origen falangista (dos o, como mucho, tres) eran disidentes deprimidos, de aspecto taciturno, como si hubieran perdido la guerra. (Santiago Ramentol, 1968-1972)

Igualmente, Carles Guárdia (1970-74) considera que en los años 70 los objetivos doctrinales de la EOP en Barcelona ya no se cumplían: "Si el objetivo era orientarnos políticamente fue un fracaso, ya fuese por incompetencia o desprestigio de los que deberían hacerlo o bien porque la mayoría del alumnado era ya muy refractario".

Manel López (1970-74) extiende esa permisividad incluso a la dirección de la Escuela en la capital catalana: "La dirección era pro régimen, pero en aquellos momentos sabía que había empezado el cambio de ciclo y se mostraba elegantemente condescendiente con nuestras peticiones".

En el caso de la Sección de la EOP de La Laguna (1963-1975) los alumnos coinciden en recordar la Escuela como un ámbito de insólita libertad en medio de un contexto autoritario y represor. Una visión que ya había expuesto Pestano (2010) que atribuía esa situación a la lejanía de Madrid y al hecho de que la sección lagunera se pusiese en funcionamiento en los años 60 (1963) "en los que se constataba el debilitamiento del pensamiento falangista" (p. 571).

Julio Pérez (1968-72) define el ambiente que se vivía en La Laguna como "especialmente abierto, tolerante y progresista" y señala que el clima no era de afinidad al régimen franquista "sino más bien de hostilidad". Eso sí, una hostilidad que describe como cuidadosa y prudente, tal y como se manifestaban las políticas contrarias a la dictadura en esa época.

Ninguno de los tres encuestados que estudiaron en La Laguna recuerda haber recibido adoctrinamiento ideológico por parte de los profesores ni por la dirección del centro. Juan Cruz es el más categórico (1967-1970): "Los profesores jamás nos dieron una consigna. Ni siquiera implícitamente". En el mismo sentido, Max Ebstein rechaza que existiese en los docentes un afán de "domesticar" en el régimen a los futuros periodistas: "Los tres años de 
estudios en la EOP no tuvieron ni un momento de presión o comentario político y en cambio excelentes profesores y periodistas de gran categoría".

Esa relativa laxitud ideológica contrasta con la dureza de la censura que los alumnos encontraron una vez que se incorporaron a la profesión al salir de la Escuela. Son varios los encuestados que evidencian la fiscalización opresiva que los periodistas sufrieron en su ejercicio profesional. Luis María Ansón (1954-1957) define la censura de la época como "atroz". Expresión similar emplea Santiago Ramentol (1968-1972) que la tilda de "asfixiante". Antonio Merino (1968-1972), por su parte, subraya la paradoja que se daba entre el ambiente de la Escuela y la realidad que vivían los periodistas en los medios: "En las prácticas la censura actuaba con precisión. Profesores que eran liberales en la Escuela actuaban como censores en los medios".

\subsubsection{Visión profesionalizante: una formación para el "oficio" de periodista}

Los exalumnos de Madrid encuestados consideran que los estudios recibidos fueron de relativa calidad, incluso, para algunos entusiastas, equiparables a los estudios universitarios, con profesores en su mayoría cualificados, y entienden que la formación que recibieron les capacitó para ejercer la función de periodistas con solvencia. Esta visión formativa se corresponde con una época en la que el Periodismo, "equivocadamente se consideraba más un oficio que se aprende con la práctica que una profesión regulada y científica" (entrevista a Aguinaga, 2018). Tal vez por ello en la Escuela "predominó la práctica y la visión profesional, no tanto académica y 'cientifista'” (entrevista Aguinaga, 2018).

Luis Ángel de la Viuda (1956-1959) considera que la Escuela formaba adecuadamente para el ejercicio del periodismo gracias a que había prácticas en medios de comunicación y que "los profesores eran periodistas y muy buenos". Diego Carcedo (1965-1969) destaca en el mismo sentido que la Escuela "ofrecía el contacto con profesores que eran profesionales y mayor relación con los medios (...). Se garantizaban las prácticas en los veranos y eso hacía que se saliese con un conocimiento técnico superior al actual".

Al mismo tiempo, son varios los exalumnos que aluden a las deficiencias de la Escuela en lo que se refiere a equipamiento técnico que impedía una formación más completa: "Eran otros tiempos mucho más rudimentarios y menos exigentes en el ejercicio de la profesión. Los profesores hacían lo que podían y con escasez de medios técnicos" (Jesús Picatoste, 19601964).

Una parte destacada de los profesores de la Escuela de Madrid son recordados como verdaderos maestros de Periodismo. Muchos eran directores de medios o periodistas en ejercicio, por lo que ofrecían una formación cercana a la realidad de la prensa. Los exalumnos entrevistados destacan a docentes tales como Enrique de Aguinaga (redactor jefe y subdirector de Arriba), Pedro Gómez Aparicio (director de la Agencia EFE y de la Hoja del Lunes), Emilio Romero (director de La Mañana, Información y Pueblo) o Bartolomé Mostaza (subdirector de Arriba y director de Ya).

Sin embargo, también en este aspecto se presentan claras diferencias entre la Escuela de Madrid y las sedes de Barcelona y La Laguna. Los alumnos de Barcelona se muestran mucho más críticos con la calidad de las clases de las que destacan las asignaturas de formación humanística dictadas por catedráticos universitarios de diferentes áreas, frente a las propias 
del Periodismo que critican por su amateurismo e improvisación: "Hubo asignaturas, profesores y momentos interesantes. Pero el plan de estudios tenía un enfoque de formación profesional básica. Y los estudios eran, además, muy caóticos: una asignatura por ahí y otra por allá con escasa coherencia" (Santiago Ramentol, 1968-1972). Carles Guardia (1970-1974) señala la gran diferencia existente entre los profesores que impartían las asignaturas de cultura general (Historia, Economía, Lengua) que sobresalían por su calidad, frente a los docentes de las asignaturas propias de la práctica profesional que describe como "muy flojos".

Situación similar se daba en la delegación de La Laguna. Juan Cruz (1967-1970) detalla la falta de organización de las clases que parecían más bien "charlas con los profesores" sobre el oficio.

Eran más bien coloquios, intercambio de experiencias, gente que trabajaba en otra cosa enseñando a gente que estaba pensando en otra cosa. Sólo había la voluntad, compartida, de aprender, pero nada fue sobresaliente, o nada quedó como sobresaliente en mi memoria.

\subsubsection{Transición a los estudios universitarios de Periodismo}

Para la mayoría de los entrevistados, la Escuela constituyó un impulso más que una rémora a la entrada del Periodismo en la Universidad, pero se cuestiona si su existencia misma impidió que el tránsito se produjese antes. Aguinaga entiende la Facultad de Ciencias de la Información de la Universidad Complutense como la continuación de la EOP: "La Escuela dio a la Universidad profesores, métodos y materias" (entrevista, 2018). Merino considera la Escuela como el "embrión" de las nuevas Facultades de Comunicación. Para Ansón, el impulso realizado desde dentro de la propia Escuela resultó clave para conseguir la incorporación del Periodismo a la Universidad, una labor en la que él mismo destacó junto con el último director de la EOP Emilio Romero.

Otros encuestados, conceden menos protagonismo a la Escuela en ese tránsito y entienden que el paso a la Universidad se produjo de forma natural: "Ya estaba en muchos países (la carrera de Periodismo) y no tenía sentido mantenerla en el ámbito de una Escuela Oficial que, aunque prestaba formación, fundamentalmente respondía a objetivos políticos" (Diego Carcedo, 1965-1969). Para Santiago Ramentol (1968-1972) la formación preuniversitaria, tanto la oficial como la proporcionada por las escuelas de la Iglesia, había de desembocar necesariamente en los estudios superiores.

Aquellas escuelas forjaron todo tipo de periodistas: buenos, malos y mediocres. En general, demócratas. Cuando se agotaron sus recursos y objetivos, cedieron el paso a unos estudios de rango universitario, lo cual era, dada la función social de la información, un paso necesario, imprescindible.

Más directa es la vinculación Escuela-Universidad en la sección de La Laguna. De hecho, La Laguna se arroga ser la primera universidad pública que acogió los estudios de Periodismo en España. Julio Pérez (1968-1972) reconoce que ese carácter ya se percibía en la Escuela.

La Escuela era un centro universitario más, su secretaría y su administración y sus aulas eran físicamente las de un centro más (...) El precedente de la Escuela se invocó para reclamar la implantación ulterior de los estudios de Ciencias de la Información. 
De hecho, algunos de los estudiantes entrevistados se convirtieron con el tiempo en profesores de las primeras universidades que impartieron la Licenciatura de Periodismo a partir de 1971. Es el caso de Enrique de Aguinaga, Amparo Moreno Sardá o Santiago Ramentol.

El salto a la enseñanza superior no acabó, sin embargo, con el debate académico en torno al rango de estudios que requieren los periodistas. Así relata Aguinaga las dudas con las que esta titulación fue recibida en la Universidad:

En principio se iba a llamar Facultad de Periodismo, pero la llamaron Ciencias de la Información, y los contrarios argumentaban que no se hablaba de Ciencias de la Medicina o Ciencias del Derecho.... En realidad, esta circunstancia es una prueba más de que la universidad nos acogió malamente, éramos de segunda clase. La universidad nos recibió como intrusos (entrevista a Aguinaga, 2018).

Una visión que, de manera similar, menciona Amparo Moreno (1970-1973) desde Barcelona.

Habría que analizar hasta qué punto la entrada del Periodismo en la Universidad ha estado marcada por el lastre de los prejuicios académicos contra el periodismo y la cultura de masas (...). Este lastre del pensamiento académico ha dificultado que, una vez los estudios de Periodismo en la Universidad, ésta se haya enriquecido con sus aportaciones. Al contrario, ha impuesto sus reglas y con frecuencia ha impedido la apertura a los nuevos interrogantes y enfoques más amplios que requiere el mundo de los medios de comunicación.

Al contrario que muchas otras titulaciones, los estudios de Periodismo "no se crean en la Universidad; se incorporan a la Universidad" ${ }^{5}$. Un origen y un pasado que permiten entender la evolución de esta titulación en la actualidad.

\section{DISCUSIÓN Y CONCLUSIONES}

La dictadura franquista (1939-1975) ejerce un control informativo a través de la censura, un control formativo a través de la Escuela Oficial de Periodismo (EOP) y un control profesional a través del Registro Oficial de Periodistas. En este contexto, las aportaciones de la EOP a la formación de periodistas en España forman parte de un debate académico sin cerrar. Coincidiendo con la tesis de Aguinaga (1984), la exigencia de titulación y colegiación representa por sí misma el primer intento serio en España de regular profesionalmente el Periodismo mediante una normativa laboral y es la Escuela la que viene a "crear la titularidad del periodista" (1984, p.138). Pero, al mismo tiempo, ejerce su papel censor y de control de la profesión (Humanes, 1997; Videla 2002; Real, 2004) a través del registro formativo y colegial de los periodistas afines al Régimen.

Esta visión dicotómica, sin embargo, no puede entenderse de la misma forma durante los 30 años de historia de la EOP, por lo que esta investigación confirma la necesidad de analizar su influencia formativa por etapas diferenciadas. La EOP efectivamente utilizó la formación de los periodistas como una vía de control y censura informativa 'preventiva' unida a la colegiación obligatoria que dejaba al margen a los periodistas no afines. Pero este estudio refleja también

\footnotetext{
${ }^{5}$ Discurso de Aguinaga sobre la EOP en el XXV aniversario de la Facultad de Ciencias de la Información de la Universidad Complutense, reivindicando el papel de la Universidad (discurso inédito cedido por el autor)
} 
cómo ese control férreo fue relajándose durante sus tres décadas a través de las sucesivas normativas y la apertura hacia otras iniciativas formativas agrupadas aquí en tres etapas.

La primera etapa de control férreo formativo y colegial (1941-1958) está marcada por la necesidad acuciante del Régimen de conseguir inscritos afines a su recién estrenado Registro Oficial, pero estableciendo filtros que alejaran a "cualquier elemento ajeno al sistema" (Videla, 2002, p.146-147).

La segunda etapa se considera de expansión y aperturismo formativo (1958-1966) por el aumento de centros y la ampliación de los programas. Se estrenan dos escuelas privadas (el Instituto de Periodismo de Navarra en 1958 y la Escuela de Periodismo de la Iglesia) y la EOP abre dos delegaciones en Barcelona y la Laguna (1964), centros que van alcanzando mayor independencia favorecidos por el aperturismo de la Ley de Prensa de 1966.

La tercera etapa pre-universitaria (1969-1975) constituye la de mayor despolitización de la EOP. La consideración de los autores es unánime al señalar que al programa se le resta ideología en favor del carácter formativo (Videla, 2002; Real, 2004; Aguinaga, 2018). La titulación universitaria de Periodismo es aprobada en 1970 y es la Universidad Complutense la que hereda parte del programa y del profesorado de la EOP que cierra en 1975.

Existe, pues, una clara evolución interna de la propia Escuela, tal como corroboran también los exalumnos entrevistados de Madrid, Barcelona y La Laguna, que acusan diferencias en sus respectivas sedes. De sus testimonios se concluyen varias ideas concretas.

Respecto a la influencia ideológica de la EOP, los alumnos coinciden en que nació marcada por "directrices políticas" para identificar a periodistas y con profesores afines al Régimen. Aun así, la mayoría de los encuestados -cuya experiencia se corresponde principalmente con las dos segundas etapas- no recuerda haber recibido presiones ideológicas expresas en las clases. Algunos señalan como fracasado el intento de formación ideológica en el aula porque, frente al ambiente exterior de censura 'asfixiante', en la Escuela se describe un ambiente de alumnos cada vez más críticos.

En relación con la percepción formativa, los alumnos señalan una relativa calidad, aunque con consideraciones dispares. Desde la delegación de Madrid destacan como valiosos a determinados profesores y la capacitación práctica que permitía aprender a ser periodista. Desde las aulas de Barcelona y la Laguna se muestran más críticos al destacar el valor de la formación humanística frente el amateurismo de la enseñanza profesional.

Por último, en cuanto al debate acerca de si la EOP fue rémora o impulso para la enseñanza universitaria del Periodismo, la mayoría interpreta que supuso un impulso y varios de los encuestados recuerdan espontáneamente a Ansón y Romero como los artífices finales. Otros alumnos consideran el fin de la EOP como natural y necesario por sus propias limitaciones formativas y por la estela internacional. Cabe destacar, cómo algunos encuestados reflejan que los reparos para dar el salto a la enseñanza superior venían de la propia Universidad, reticente a acoger los estudios de Periodismo por no considerarlos conocimiento científico.

En definitiva, tanto la revisión documental como la consulta a fuentes primarias permiten afirmar que el control ideológico y la calidad formativa de la EOP experimentan una evolución interna en diferentes etapas paralelas al contexto político y social en el que primó la política informativa sobre el debate formativo (Humanes, 1997). De hecho, si hay que considerar algún 
tipo de lastre es el de la tardanza en la consideración del Periodismo como área de conocimiento dentro de la propia Universidad. Un debate inacabado en torno a la adecuada formación de los periodistas que se arrastra incluso hasta hoy en un entorno científico consolidado.

\section{Referencias}

ABC (2 de mayo de 1970). El Periodismo a la Universidad. Entrevista a Emilio Romero, 11-14.

Acirón, R. (1988). La Facultad de Ciencias de la Información. En Historia de la Universidad de La Laguna. Servicio de Publicaciones de la ULL, 3(2), 109-163.

Aguinaga, E. (1984). Epistemología del ejercicio periodístico. Los estudios de periodismo y su proyección profesional [Tesis inédita, Universidad Complutense de Madrid].

Aguirre, M. (1988). El deber de formación en el informador. Eunsa.

Altabella, J. (1971). Historia del Periodismo. Programa Escuela Oficial de Periodismo, 1971-1972. Escuela Oficial de Periodismo.

Altabella, J. (1979). Breves notas para una historia de la formación del periodista en España. Asociación de Editores de Diarios Españoles, AEDE(2), 34-39.

Araujo, F. (1899). La primera Escuela de Periodismo. La España Moderna, 128, 147-151.

Aróstegui, J. (1995). La investigación histórica: Teoría y método. Crítica.

Baldó Lacomba, M. (2008). La universidad española bajo el franquismo y contra el franquismo (19391975). En de Lourdes Alvarado, M. \& Pérez Puente, L. (coords.) Cátedras y catedráticos en la historia de las universidades e instituciones de educación superior en México. III. Problemática universitaria en el siglo XX (pp. 35-69). IISUE-UNAM. https://bit.ly/39CUtJT

Baldó Lacomba, M. (2016). Regeneración universitaria y movimiento estudiantil en España. En Hidalgo Prego, M. \& Ríos Zúñiga, R. (coords.) Poderes y educación superior en el mundo hispánico: siglos XV al XX (pp. 453-474). IISUE-UNAM. https://bit.ly/3m0CeUH

Barrera, C. (2002a). Notas para una historia del Instituto de Periodismo de la Universidad de Navarra (1958-1971). Comunicación y Sociedad, 15(1), 7-38. https://bit.ly/3u8J0eF

Barrera, C. (2002b) La apertura informativa como elemento configurador de la prensa del tardofranquismo. En: J.A. García Galindo, J.F. Gutiérrez Lozano \& I. Sánchez Alarcón (Eds.), La comunicación social en el franquismo. Centro de Ediciones de la Diputación Provincial de Málaga, 411-427. https://bit.ly/3EQf1NI

Beneyto, J. (1944). Planteamiento del régimen jurídico de Prensa y Propaganda. Instituto Editorial.

Beneyto, J. (1957). El periodismo como técnica y como cultura. Escuela Oficial de Periodismo.

Beneyto, J. (1958). La formación de periodistas. Estudio mundial sobre la preparación del personal de la información. UNESCO. http://unesdoc.unesco.org/images/0013/001353/135346so.pdf

Benito, A. (1967). Evolución de los estudios de Periodismo en el mundo. En: Ciencia y enseñanza del Periodismo. Cuadernos de Trabajo, 11, 13-36.

Díaz Sánchez, P. y Gago González, J.M. (2006). La construcción y utilización de las fuentes orales para el estudio de la represión franquista. Revista de Historia Contemporánea, 6, 1-25. https://bit.ly/3EMfznA

Fernández del Moral, J. (1991). Información Periodística Especializada. En: A. Benito. (Coord.) Diccionario de Ciencias y Técnicas de la Comunicación. Ediciones Paulinas, 764-784.

Gordon, M. (1991). La enseñanza del Periodismo en el mundo occidental. Estudio Comparado de tres escuelas [Tesis doctoral, Universidad Complutense de Madrid].

Humanes, M. L. (1997). La formación de los periodistas en España [Tesis doctoral, Universidad Complutense de Madrid]. 
Humanes, M. L. (2002). La política de formación de comunicadores: la Escuela Oficial de Periodismo (1941-1970). En: J.A. García Galindo; J.F. Gutiérrez Lozano \& M.I. Sánchez Alarcón (coord.). La comunicación social durante el franquismo. Diputación Provincial de Málaga, 625-642. https://bit.ly/3o60zJp

Luján, L. (2014). La política informativa tardofranquista. El modelo de control de la empresa privada de prensa, (1966-1975) [Tesis doctoral, Universidad Complutense de Madrid].

Mainar, R. (2005). El arte del periodista. Destino.

Moragas, M. (1981). Teorías de la Comunicación. Gustavo Gili.

Núñez, M. (1997). El ojo de la aguja. El carnet de periodista, el último filtro de la depuración profesional en la inmediata posguerra. Historia y Comunicación Social, 2, 205-210. https://bit.ly/3o5a91d

Pestano, J. M. (2010). La formación de periodistas en Canarias durante el tardofranquismo. La sección de la Escuela Oficial de Periodismo de la Universidad de La Laguna (1963-1975). En Yanes Mesa, J. A. (coord.), El periodismo y la cohesión territorial del archipiélago. Real Sociedad Económica de Amigos del país de Tenerife. https://bit.ly/3ube4ud

Pestano, J. M., Rodríguez-Wangüemert, C. y Del Ponti, P. (2011). Transformaciones en los modelos de formación de periodistas en España. Estudios sobre el Mensaje Periodístico, 17(2), 401-415. https://doi.org/10.5209/rev_ESMP.2011.v17.n2.38122

Ramos Pérez, G. y Studer Villazán, L. (2012). La fuente oral en la recuperación de la memoria histórica: el caso de los desaparecidos de Tenerife. En Acosta Guerrero, E. (coord.) XX Coloquio de Historia Canario-Americana (pp. 1150-1160). Cabildo Insular de Gran Canaria. https://bit.ly/3zCaF8R

Real, E. (2004). Formación y ejercicio profesional del periodista en la España del siglo XXI dentro del marco de la Unión Europea [Tesis doctoral, Universidad Complutense].

Rodrigo, M. (2001). Teorías de la Comunicación. Ámbitos, Métodos y Perspectivas. Universidad Autónoma de Barcelona.

Salaverría, R. y Barrera, C. (2009). The Spanish Journalism Education Landscape. En: G. Terzis (Ed.) European Journalism Education. Intellect, 319-330.

Sánchez-García, P. (2014). Un siglo de enseñanza periodística en España: de la primera escuela de Periodismo a la adaptación de los estudios al Espacio Europeo de Educación Superior [Tesis doctoral, Universidad de Valladolid].

Sánchez-García, P. (2016). Los efectos de la primera fase del EEES en la enseñanza del Periodismo en España: mayor especialización y formación práctica. Communication \& Society, 29(1), 125-143. https://doi.org/10.15581/003.29.1.sp.125-143.

Sánchez-García, P., García-Orosa, B.; López-García, X. \& Vázquez-Rodríguez, A. (2019). Perfiles periodísticos emergentes reconocidos en la Universidad: investigación, conceptualización y oferta en el Grado. Trípodos, 45, 157-177. https://doi.org/10.51698/tripodos.2019.45p157-177

Sanz Hernández, A. (2005). El método bibliográfico en investigación social: potencialidades y limitaciones de las fuentes orales y los documentos personales. Asclepio, 57(1), 99-115. https://doi.org/10.3989/asclepio.2005.v57.i1.32

Sevillano Calero, F. (1998). Propaganda y medios de comunicación en el Franquismo. Publicaciones de la Universidad de Alicante.

Tejedor, S. (2006). La enseñanza del ciberperiodismo en las licenciaturas de periodismo en España [Tesis doctoral, Universidad Autónoma de Barcelona].

Videla, J. J. (2002). La formación de los periodistas en España: perspectiva histórica y propuestas de futuro [Tesis doctoral, Universidad Complutense de Madrid].

Vigil y Vázquez, M. (1987). El Periodismo enseñado. De la Escuela de "El Debate» a Ciencias de la Información. Mitre. 


\section{Semblanza de las autoras}

Dra. Pilar Sánchez-García es profesora en la Universidad de Valladolid y miembro del grupo de investigación Nuevas Tendencias en Comunicación (NUTECO). Participa en dos proyectos competitivos de ámbito nacional. Cuenta con una treintena de publicaciones centradas principalmente en tres temáticas: la evolución de la enseñanza periodística, los perfiles profesionales y la narrativa multimedia. Es autora del monográfico Periodistas (in)formados. Un siglo de enseñanza periodística en España: historia y tendencias (2017).

Dra. Marta Redondo es profesora de la Universidad de Valladolid. Es miembro del grupo de investigación Nuevas Tendencias en Comunicación (NUTECO). Participa en varios proyectos de ámbito nacional. Su investigación se centra en el análisis del sensacionalismo mediático, la ética periodística, el infoentretenimiento y la comunicación política. Es autora o coautora de una treintena de capítulos de libro y artículos. Es Premio Extraordinario de Doctorado y Premio Internet de Periodismo de Castilla y León.

Alba Diez-Gracia es doctoranda y graduada en Periodismo y en el Máster de Investigación de la Comunicación como Agente histórico-social de la Universidad de Valladolid, en el que obtuvo el Premio Extraordinario. Integrante del Proyecto de Innovación Docente LabCom UVa y del Proyecto Politainment in the face of media fragmentation. Su investigación se centra en la evolución de la agenda setting y en el análisis del entorno digital y sus audiencias. 